\section{Mixoma odontogénico, presentación de caso y revisión de literatura}

\section{Odontogenic myxoma, case report and literature review}

\section{Resumen}

El mixoma odontogénico es una lesión benigna que se presenta mayormente entre la segunda y cuarta década de vida, con mayor frecuencia en zona de molares y mayor incidencia en hombres que en mujeres (2:1). Histológicamente presenta células estrelladas y fusiformes dispersas en una matriz de aspecto mixoide muy abundante. El objetivo de este artículo es reportar un caso clínico de un mixoma odontogénico, así como una breve revisión de la literatura. Una paciente mujer de 39 años que acudió a la clínica de Cirugía Oral y Maxilofacial por presentar aumento de volumen localizado en mandíbula en zona vestibular entre dientes 31 y 44, asintomática a la palpación, con una evolución de aproximadamente doce meses. Se decidió realizar una biopsia incisional para el estudio histopatológico. El resultado del estudio histopatológico fue mixoma odontogénico por lo que se realizó el tratamiento de resección con márgenes libres y colocación de placa de reconstrucción, así como controles posoperatorios.

Palabras clave: Tumor odontogénico; Mixoma; Biopsia; Patología; Mandíbula (fuente: DeCS BIREME).

\begin{abstract}
The odontogenic myxoma is a benign lesion that occurs mostly between the second and fourth decade of life, more frequently in the molar area and with a higher incidence in men than in women (2:1). Histologically, it presents starred and fusiform cells dispersed in a very abundant myxoid matrix. The objective of this article is to report a clinical case of an odontogenic myxoma, as well as a brief review of the literature. Subject: a 39-year-old female patient who attended the Oral and Maxillofacial Surgery clinic due to an increase in volume located in the jaw in the vestibular area between teeth 31 and 44; asymptomatic at palpation, with an evolution of approximately twelve months. An incisional biopsy was performed for the histopathological study. The result of the histopathological study was an odontogenic myxoma, so we decided that the best treatment was a free margin resection and reconstruction plate placement.
\end{abstract}

Keywords: Odontogenic tumors; Myxoma; Biopsy; Pathology; Mandible (source: MeSH NLM).

\section{Caso Clínico}

James Jerez Robalino ${ }^{1, a}$, Fabiola Salgado Chavarría ${ }^{1, b}$, Ernesto Lucio Leonel ${ }^{1, b}$, Sergio Olmedo Cueva $1, b$

${ }^{1}$ Universidad Nacional Autónoma de México, Facultad de Odontología, Especialidad de Cirugía Oral y Maxilofacial, División de Estudios de Postgrado e Investigación, Ciudad de México, México.

${ }^{a}$ Cirujano Dentista.

${ }^{\mathrm{b}}$ Especialista en Cirugía Oral y Maxilofacial.

\section{Correspondencia:}

James Philippe Jerez Robalino: jamje15@hotmail.com

Copilco Universidad, Cerro Culiacán 19, Coyoacán,

Ciudad de México, México.

ORCID: 0000-0002-3441-424X

\section{Coautores:}

Fabiola Salgado Chavarría: cmf_fabiolasalgado@hotmail.com ORCID: 0000-0002-8507-8346

Ernesto Lucio Leonel: eluciol@enes.unam.mx ORCID: 0000-0002-1910-8287

Sergio Olmedo Cueva: sergio85olmedo@gmail.com ORCID: 0000-0003-1288-9954

\section{Editora:}

Marieta Petkova-Gueorguieva

Universidad Nacional Mayor de San Marcos, Perú.

Conflicto de intereses: no se presentaron conflictos de interés durante la realización de este trabajo.

Fuente de financiamiento: autofinanciado.

Recibido: $15 / 02 / 20$

Aceptado: $17 / 05 / 20$

Publicado: 04/08/20 


\section{Introducción}

El mixoma odontogénico (MO) es una neoplasia benigna caracterizada por células estrelladas y fusiformes dispersas en abundante matriz extracelular mixoide ${ }^{1}$. Virchow en 1863 introdujo el término de mixoma para un grupo de tumores de histología semejante a la sustancia mucinosa del cordón umbilical ${ }^{2}$. El MO fue descrito por primera vez por Goldman y Thoma en $1947^{2}$. Se observa una mayor incidencia del MO en zonas dentadas de la mandíbula, en ocasiones asociado con dientes no erupcionados, pero su presencia siempre guarda relación con tejido dental presente en la zona donde aparece ${ }^{3}$. Es el tercer tumor odontogénico más común (después del odontoma en primer lugar y ameloblastoma en segundo) ${ }^{4}$, la mayoría de los casos se presentan entre la segunda y cuarta década de la vida ${ }^{5}$.

Estas lesiones representan el 3-6\% de todos los tumores odontogénicos, son usualmente asintomáticas y se encuentran incidentalmente en radiografías, las patologías no son encapsuladas, lo que permite la infiltración substancial en el hueso medular adyacente ${ }^{6}$. El MO tiene un crecimiento lento, puede provocar expansión de las corticales y su naturaleza agresiva causa perforación ósea, resorción de raíces dentales, desplazamiento y movilidad dental ${ }^{7}$.

El patrón imagenológico del tumor de apariencia de pompas de jabón, raqueta de tenis o panal de abeja se asemeja a un ameloblastoma, por lo que se debe realizar una biopsia excisional en lesiones pequeñas e incisional en lesiones grandes que abarquen grandes áreas en el hueso ${ }^{4}$. También es recomendable realizar una adecuada diferenciación de otras neoplasias similares por sus características clínicas, radiográficas e histológicas en la mandíbula como el tumor odontogénico primordial, ameloblastoma, fibroma condromixoide, el neurofibroma mixoide y en maxilar de pólipos nasales ${ }^{4,6}$.
El tratamiento para esta patología es la enucleación y curetaje en lesiones que no abarcan grandes cantidades de estructura ósea (de tres centímetros o menos), obteniéndose resultados satisfactorios sin recurrencias a largo plazo ${ }^{3}$. Cuando las lesiones son más amplias, se prefiere resecciones en bloque dejando los bordes libres de la lesión para disminuir la recurrencia reportada. En los tratamientos en general, entre un $10-33 \%$ con un promedio de recurrencia del $25 \%$; por ello se debe tener un margen de seguridad de $1,5 \mathrm{~cm}$ (debido a la invasión de hueso trabecular) ${ }^{3}$.

El propósito de este reporte de caso clínico es mostrar los resultados que se obtienen con una resección en bloque con márgenes libres a los doce meses de seguimiento ya que en este tipo de patologías que abarcan una amplia extensión el objetivo principal, que se quiere obtener, es disminuir al mínimo la posibilidad de reaparición de la patología y una reintervención.

\section{Reporte del caso}

Se presentó paciente de 39 años de sexo femenino a la clínica de Cirugía Oral y Maxilofacial de la Universidad Nacional Autónoma de México (UNAM), con un aumento de volumen localizado en mandíbula, entre el primer molar inferior derecho hasta el segundo molar inferior izquierdo, de aproximadamente un año de evolución, donde se observó que expandía corticales. Los antecedentes patológicos personales y heredo-familiares fueron interrogados y negados.

Durante la revisión clínica de la paciente, se detectó un incremento de volumen en el tercio inferior izquierdo de la cara en relación a los tejidos blandos adyacentes (coloración e hidratación de mucosas normales), higiene oral y apertura bucal adecuada (Figura 1), presentando restos radiculares de los primeros y segundos molares mandibulares. En el examen radiológico (ortopantomografía)

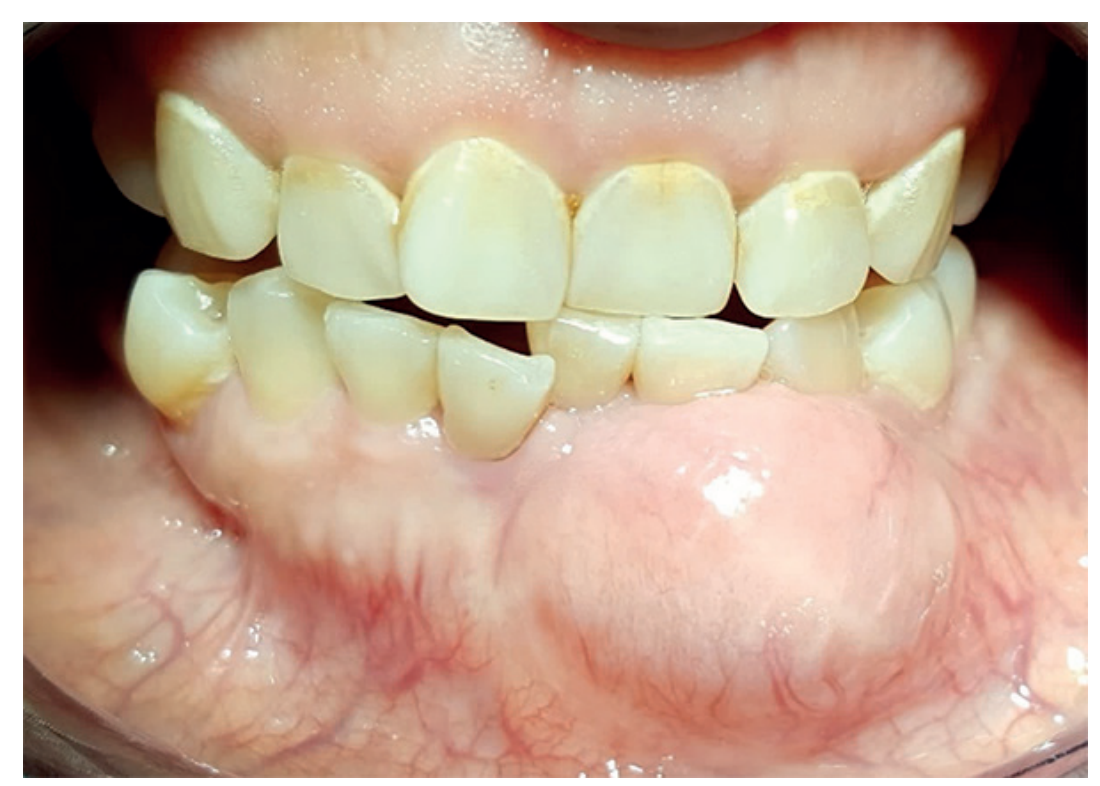

Figura 1. Fotografía intraoral en oclusión donde se observa un aumento de volumen entre los dientes 41 y 34 con coloración de mucosa sin cambios, de superficie lisa, de base sésil 
se observó una imagen radiolúcida multilobulada en la zona de sínfisis y parasínfisis desde mesial del segundo molar inferior izquierdo a mesial del primer molar inferior derecho, sugestiva de osteólisis y rizólisis. Tomográficamente (cone beam) se observaron zonas hipodensas a nivel de sínfisis, parasínfisis y cuerpo mandibular hasta mesial del segundo molar inferior izquierdo y mesial del primer molar inferior derecho (Figura 2).

Después de informar a la paciente y haber firmado el consentimiento informado sobre los procedimientos a realizar, considerando como diagnóstico presuntivo un mixoma versus ameloblastoma, se decidió iniciar con una punción exploratoria, con lo cual no se obtuvo muestra suficiente para su estudio, optándose por una biopsia incisional, obteniendo así un espécimen de tamaño suficiente. El resultado histopatológico mostró un fondo fibrilar donde se detectaron células de aspecto fusiforme y estelares que concuerdan con los hallazgos histopatológicos del MO (Figura 3). Así mismo en los cortes histológicos examinados se vio una proliferación de células que guardan estrecha relación con las características encontradas en los $\mathrm{MO}$, arrojando como resultado definitivo mixoma odontogénico.

Luego de examinar los hallazgos clínicos, imagenológicos (donde se evidenció un tamaño mucho mayor a 3 $\mathrm{cm}$ ) e histopatológicos (que confirmaron el diagnostico de $\mathrm{MO}$ ), se decidió que el plan de tratamiento más idóneo sería la resección y colocación de una placa de reconstrucción biangulada mandibular (sistema 2.4) predoblada y adaptada en un modelo estereolitográfico.

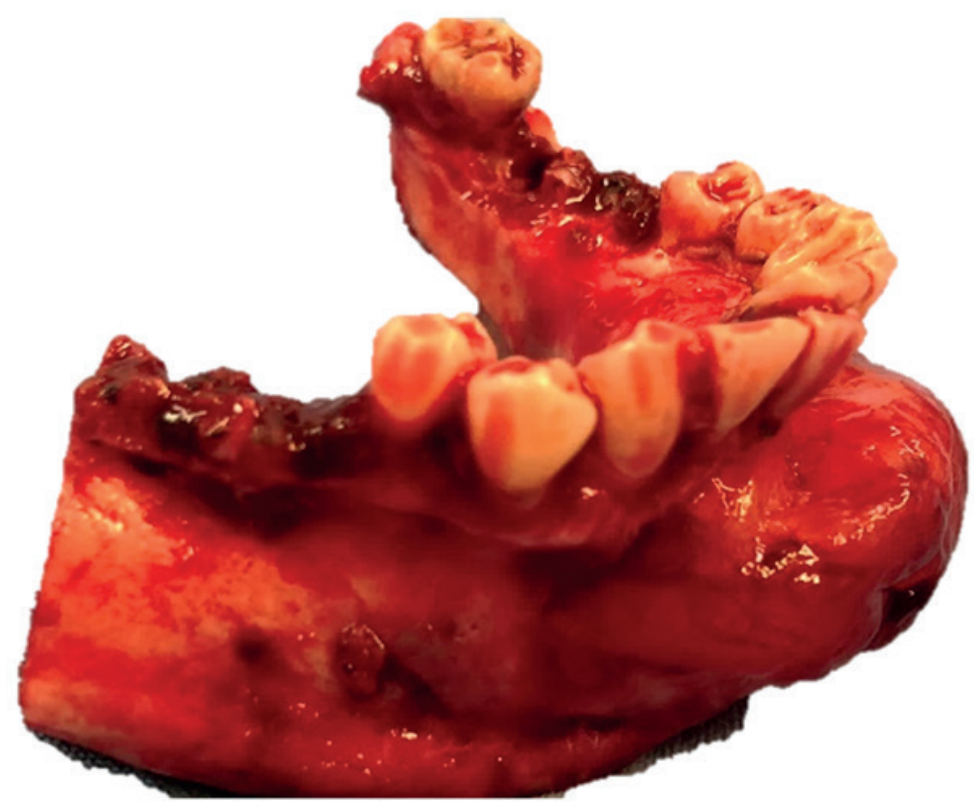

Figura 2. Espécimen obtenido de la resección quirúrgica con márgenes de $2 \mathrm{~cm}$ y enviado para su estudio histopatológico (determinando que los bordes se encontraban libres de lesión)
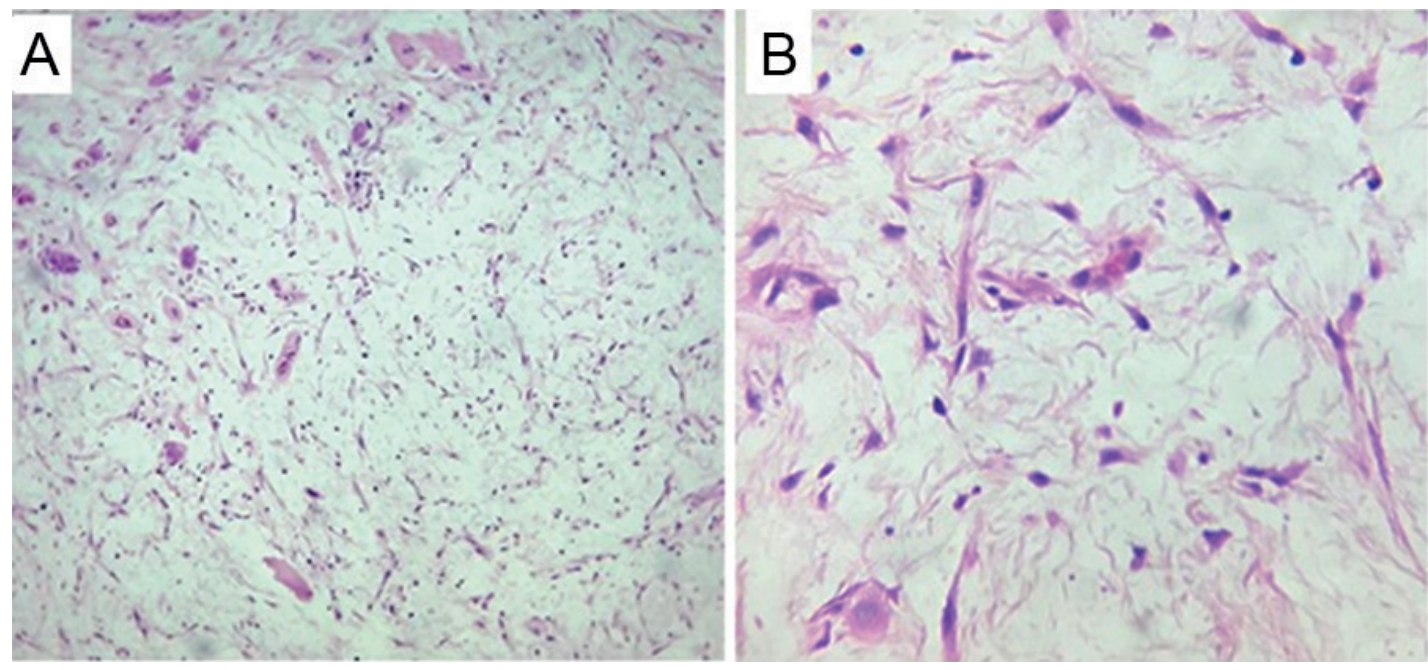

Figura 3. A. Fotomicrografía teñida con hematoxilina-eosina 40x, donde se puede observar células de aspecto fusiforme, estelar, eosinófilos polimorfonucleares, linfocitos, algunos macrófagos y células multinucleadas. B. Fotomicrografía teñida con hematoxilina-eosina 400x, mismas estructuras que en imagen (A) pero en mayor aumento 
Se realizó cirugía bajo anestesia general balanceada, procediendo a la realización del marcaje con línea punteada de la posición de la mandíbula, para evitar la lesión a estructuras de importancia como es el nervio marginal mandibular que se encuentra a $1-1,5 \mathrm{~cm}$ del borde inferior de la mandíbula según lo descrito en la literatura, y con línea continua el marcaje donde se realizaría la incisión tipo cervicotomía submandibular, incidiendo en piel, tejido celular subcutáneo, platisma, fascia cervical superficial, hasta llegar a plano óseo, exponiendo el borde inferior de la mandíbula, desinsertando de la cara interna del cuerpo mandibular la musculatura correspondiente al milohioideo, genihioideo y geniogloso, colocando punto de suspensión de la musculatura para evitar la glosoptosis y compromiso de la vía aérea.
Se procedió al marcaje sobre cuerpo mandibular para la posterior resección, se decidió colocar la placa de reconstrucción biangulada antes de realizar la resección mandibular, se colocó el sistema percutáneo ajustando los tornillos a la placa de reconstrucción, se procedió a la resección mandibular con una sierra reciprocante con irrigación abundante y protección maleable para las estructuras adyacentes, removiendo un espécimen de 13,5 x 4 x $2,5 \mathrm{~cm}$ el cual fue enviado a su estudio histopatológico (Figura 4 A y B). Se continuó con la reposición de los tejidos blandos y reconstrucción del piso de boca, suturando por planos desde el más profundo hasta el más superficial (Figura $5 \mathrm{~A}$ y B).

El resultado histopatológico, del espécimen obtenido durante la cirugía, confirmó que se identificó una patología

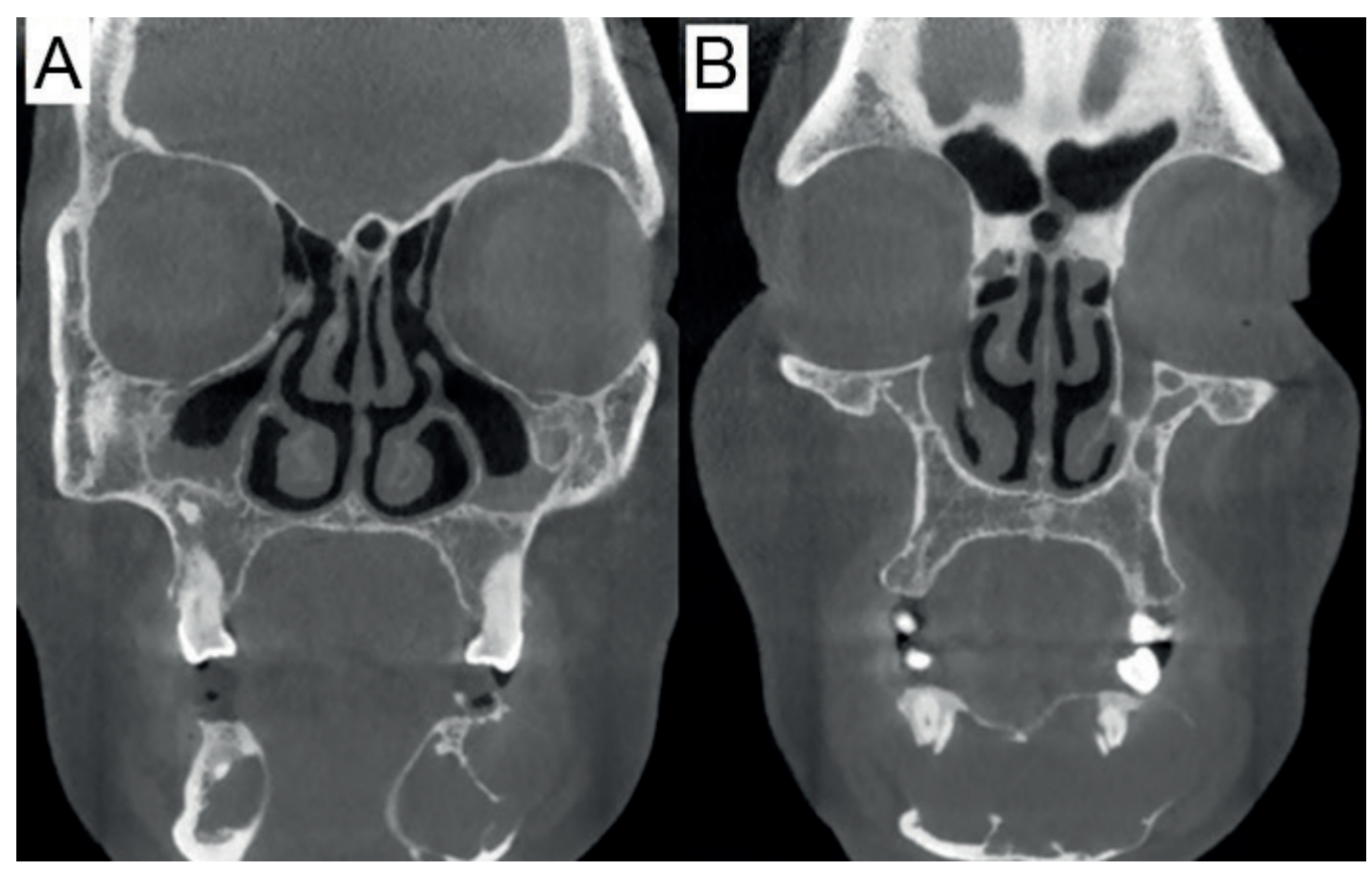

Figura 4. A. Tomografía (cone beam), de fase simple con ventana para hueso, en reconstrucción coronal, de macizo craneofacial, a nivel de primeros molares maxilares; en mandíbula se observa una zona hipodensa bilateral sugestivo de áreas osteolíticas que ocupan el cuerpo mandibular. B. Tomografía (cone beam), de fase simple con ventana para hueso, en reconstrucción coronal, de macizo craneofacial, a nivel de sínfisis y parasínfisis, en mandíbula se observa una zona hipodensa bilateral sugestivos de áreas osteolíticas que ocupa cuerpo mandibular
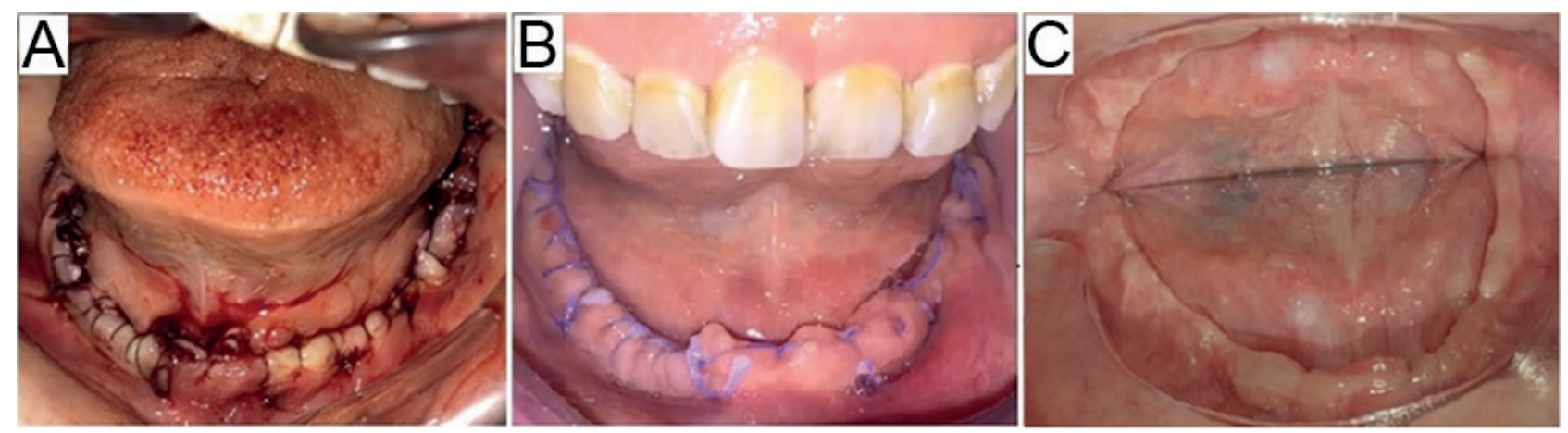

Figura 5. A. Reconstrucción del piso de boca, suturando por planos desde el más profundo hasta el más superficial, llegando a mucosa oral con puntos simples y continuos. B. Postoperatorio a las dos semanas de la cirugía donde se observa los puntos de sutura en posición adecuada ejerciendo su función. C. Postoperatorio a los 12 meses 
de estirpe mesenquimatoso compuesto por tejido fibroso laxo con matriz extracelular muy abundante basófila y aspecto mixoide, así mismo presentando espacios vasculares. Es muy importante destacar que la muestra presentó en sus extremos posteriores tejidos dentales mineralizados maduros correspondientes a dientes libres de alteración lo que nos indicaría que la posibilidad de una recurrencia es mínima y que los márgenes tomados fueron adecuados.

La paciente evolucionó satisfactoriamente en su control al año postquirúrgico, constatándose mediante ortopantomografía, áreas libres de presencia de lesión y el material de osteosíntesis en posición adecuada y cumpliendo con su función (Figura 6). La reconstrucción ósea así como el tratamiento estomatológico integral, no se pudieron realizar debido a que por motivos personales, la paciente desistió de la planificación de dichos sucesos perdiéndose por completo la comunicación. Previo a este acaecimiento, la paciente dio su asentimiento explícito para participar en esta presentación de caso clínico y su publicación.

\section{Discusión}

El MO es una lesión poco común o infrecuente de acuerdo a lo reportado en la literatura ${ }^{8-11}$. Se presenta como un tumor que deriva de elementos de tejido embrionario mesenquimal de un diente en desarrollo, incluyendo el folículo dental, papila dental o ligamento periodontal ${ }^{12,13}$.

La incidencia en Europa, Asia y América se reporta entre el $0,5-17,7 \%{ }^{14}$. Varios estudios realizados sobre el MO no definieron un grupo de edad específico para su ocurrencia, sin embargo, estos son hallados principalmente entre los 22,7- 36,9 años de edad, siendo raro en niños y en adultos mayores de 50 ańos ${ }^{8,11,12,15}$. En nuestro caso la edad de la paciente fue de 39 años, siendo el sitio afectado la mandíbula, lo que indica que ciertos casos pueden llegar a presentarse fuera de los rangos de edades reportados en la literatura.

La diferenciación con otras lesiones es una parte muy importante ya que se podría confundir el $\mathrm{MO}$ con otras neoplasias de la mandíbula como el fibroma condromixoide, el cual debe tener áreas de diferenciación cartilaginosa; el neurofibroma mixoide, el cual presenta áreas en las cuales sus células están dispuestas en fascículos desorganizados, así como células dispersas positivas para los anticuerpos dirigidos contra la proteína $s 100{ }^{1}$.

Existen dos tipos generales de tratamientos para el MO, uno conservador (curetaje, enucleación con curetaje, curetaje escisional y escisión) y también una forma no conservadora, la resección. Esta última es la que menos recurrencia reporta, pero mayor morbilidad presenta ${ }^{16,17}$. Debido que en este caso la patología tenía un tamaño considerable, lo indicado para el tratamiento fue la resección por el alto grado de recurrencia que se reporta en tratamientos conservadores en lesiones de este tamaño. Los tratamientos conservadores presentan recurrencias en promedio del $19 \%(2,1 \%-34,3 \%)$ de los casos tratados de esta forma, mientras que aquellos casos tratados por resección mostraron una tasa de recurrencia promedio del $6 \%(0 \%-17,1 \%)^{17}$.

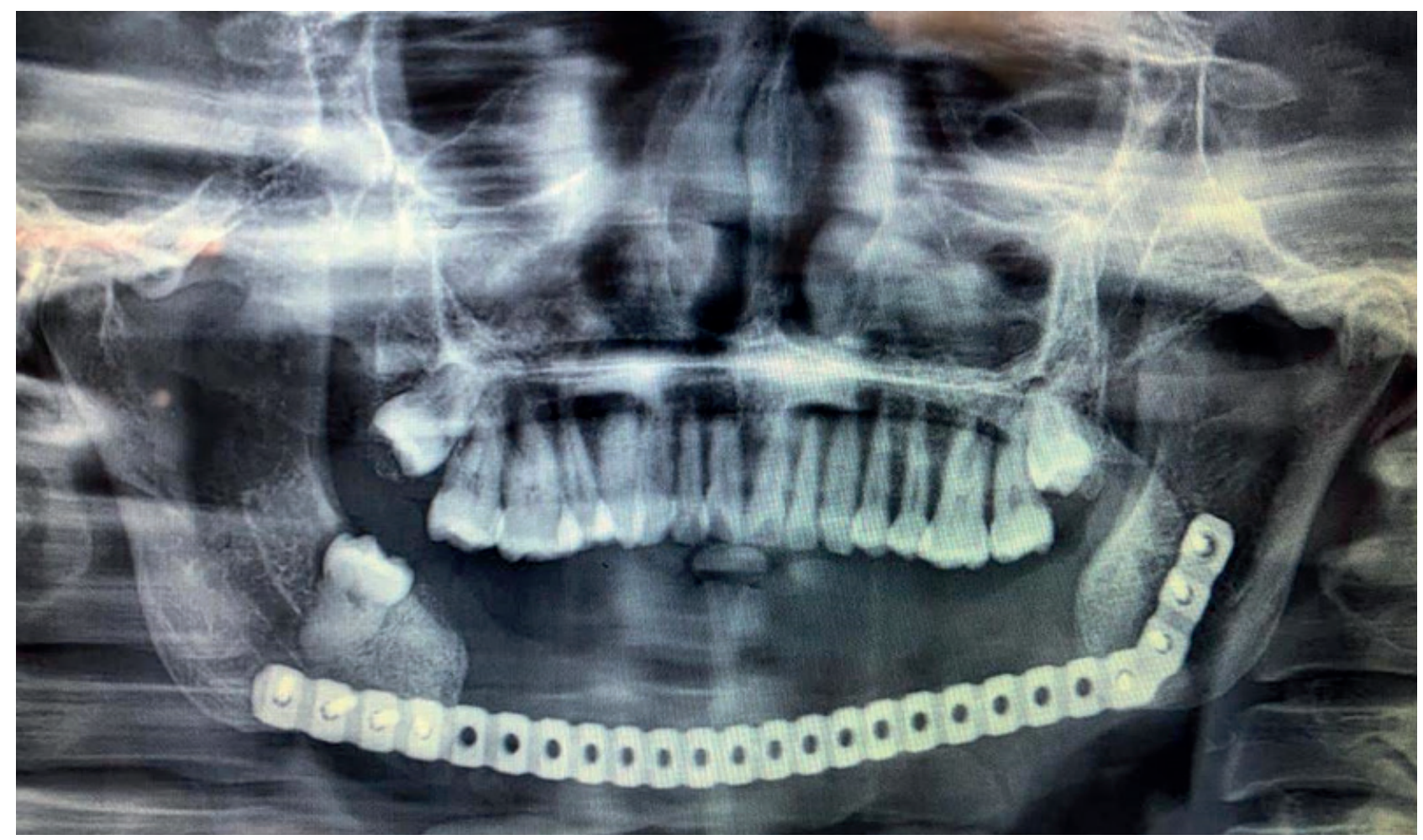

Figura 6. Ortopantomografía donde se puede constatar el área donde se realizó la resección quirúrgica, así como se observa el material de osteosíntesis utilizado que se encuentra en correcta posición y cumpliendo su función 
Se ha observado que la radioterapia por sí sola no puede erradicar esta patología, por lo tanto, el mixoma odontogénico es considerado radio resistente ${ }^{18}$. Debido a que el $\mathrm{MO}$ no está encapsulado y su infiltración produce una destrucción en el tejido duro contiguo, el riesgo de recurrencia se incrementa cuando se realizan abordajes conservadores durante su tratamiento ${ }^{19}$. Todos estos factores nos ayudan a entender el alto grado de recurrencia del $\mathrm{MO}^{5}$.

Los diferentes tratamientos son el único factor que influye en las variaciones estadísticas en cuanto a las recurrencias, por lo que se decidió realizar la resección con márgenes libres de dos centímetros con el fin de disminuir la posibilidad de recurrencia, considerando que las diferentes técnicas de tratamiento conllevarán a un mayor riesgo de reaparición de la lesión: curetaje $(31,3 \%)$, la enucleación $(13,1 \%)$, la enucleación en conjunto con curetaje $(12,7 \%)$, la enucleación y osteotomía periférica $(6,7 \%)$, la resección en bloque $(3,1 \%)$ y la resección marginal $(1,3 \%)$, siendo estos últimos tres, los tratamientos con menor grado de recurrencias ${ }^{20}$.

Las recurrencias del MO suelen ocurrir por remoción incompleta de la patología dentro de los siguientes 24 meses, aunque, esto puede acontecer mucho después 2. Presentando el caso a 12 meses de haberse realizado el tratamiento todavía se mantiene la paciente bajo seguimiento.

Es importante considerar las características tanto clínicas (tiempo de evolución, extensión y ubicación) como imagenológicas (tamaño de la lesión, unilocular o multilocular), para llegar a decidir sobre un tratamiento conservador o agresivo, procurando el menor grado de recurrencia y disminuyendo la morbilidad en los pacientes. No obstante, recordando que el estudio histopatológico es un auxiliar determinante en el diagnóstico definitivo de estas patologías.

\section{Referencias bibliográficas}

1. Neville B, Damm D, Allen C, Bouquot J. Oral and Maxillofacial Pathology. 4th Ed. Riverport Lanes, St. Louis, Missouri: Elsevier; 2016.

2. El-Naggar A, Chan J, Grandis J, Takata T, Slootweg P. WHO Classification of Head and Neck Tumours. 4th Ed. Lyon. 2017;229-230.

3. Buch SA, Babu SG, Rao K, Rao S, Castelino RL. A large and rapidly expanding odontogenic myxoma of the mandible. J Oral Maxillofac Radiol. 2017;5(1):22-6.

4. Brannon R. Central odontogenic fibroma, myxoma (odontogenic myxoma, fibromyxoma), and central odontogenic granular cell tumor. Oral Maxillofacial Surg Clin N Am. 2004;16:359-374. DOI:10.1016/j. coms.2004.03.004

5. Leiser Y, Abu-EL-Naaj I, Peled M. Odontogenic myxoma e A case series and review of the surgical management. J Craniomaxillofac Surg. 2009;37(4):206-209. DOI:10.1016/j.jcms.2008.10.001.

6. Kawase Y, Saijo H, Hoshi K, Takato T, Mori Y. Surgical management of odontogenic myxoma: A case report and review of the literature. BMC Res Notes. $2014 \mathrm{Apr}$ 5;7:214. DOI:10.1186/1756-0500-7-214.

7. Goel S, Goel M, Dinkar AD. Odontogenic mixoma of mandible with unusual (sunburst) appearance: A rare case report. J Clin Diagn Res. 2016;10(5):ZJ05-7. DOI: 10.7860/JCDR/2016/20123.7812.

8. Guzman C, Torrealba M, Kuramochi G. Odontogenic Myxoma in a Pediatric Patient: A Case Report. Open Access Library Journal. 2016;3:e3271. DOI: 10.4236/ oalib. 1103271

9. Kansy K, Juergens P, Krol Z, Paulussen M, Baumhoer D, Bruder E. Odontogenic Myxoma: Diagnostic and therapeutic Challenges in Pediatric and Adult Patients. A Case Series and Review of the Literature. J Craniomaxillofac Surg. 2012;40(7):271-276. DOI: 10.1016/j. jcms.2011.04.009

10. Lawal A, Adisa A, Popoola B. Odontogenic Tumours in Children and Adolescents: A Review of Forty-Eight Cases. Ann Ib Postgrad Med. 2013;11(1):7-11.

11. Ochsenius G, Ortega A, Godoy L, Peñafiel C, Escobar E. Odontogenic Tumors in Chile: A Study of 362 Cases. J Oral Pathol Med. 2002;31(7):415-420. DOI: 10.1034/j.1600-0714.2002.00073.x

12. Fang Q, Shi S, Sun C. Odontogenic Lesions in Pediatric Patients. J Craniofac Surg. 2014;25(3):e248-e251. DOI: 10.1097/SCS.0000000000000548

13. Thabusum D, Rajesh N, Reddy R, Ravikanth M, Raju $\mathrm{U}$. Odontogenic myxoma of maxialla- A rare case report. WJPMR 2017;3(1):282-5.

14. Khan S, Agrawal P, Sur J. A rare case report of myxoid fibroma of maxilla. Quant Imaging Med Surg 2015;5(5):778-82. 4292.2014.07.11

15. Sivakumar G, Kavitha B, Saraswathi T, Sivapathasundharam B. Odontogenic myxoma of maxilla. Indian J Dent Res. 2008;19(1):62-5.

16. Rocha A, Gaujac C, Ceccheti M, Amato-Filho G, Machado G. Treatment of recurrent mandibular myxoma by curettage and cryotherapy after thirty years. Clinics. 2009;64(2):149-52. DOI: 10.1590/S180759322009000200013

17. Saalim M, Sansare K, Karjodkar F, et al. Recurrence rate of odontogenic myxoma after different treatments: a systematic review. Br J Oral Maxillofac Surg. 2019 Dec; 57(10):985-991. DOI:10.1016/j.bjoms.2019.09.005

18. Landa L, Hedrick M, Nepomuceno-Perez M, Sotereanos G. Recurrent myxoma of the zygoma: A case report. J Oral Maxillofac Surg. 2002;60(6):704-8. DOI:10.1053/ joms.2002.33126

19. Dabbaghi A, Nikkerdar N, Bayati S, Golshah A. Rare appearance of an odontogenic myxoma in cone-beam computed tomography: A case report. J Dent Res Dent Clin Dent Prospects. 2016;10(1):65-8. DOI: 10.15171/ joddd.2016.010

20. Chrcanovic B, Gomez R. Odontogenic myxoma: an updated analysis of 1692 cases reported in the literature. Oral Diseases. 2019 Apr;25(3):676-683. DOI:10.1111/ odi. 12875 